\title{
GESTURE EN ROUTE TO WORDS: EVIDENCE FROM DANISH AND ZAPOTEC CHILDREN AND THEIR CARETAKERS
}

\author{
GESTOS EN CAMINO HACIA LAS PALABRAS: EVIDENCIAS A \\ PARTIR DE NIÑOS DANESES Y ZAPOTECOS Y SUS CUIDADORES
}

\author{
KRISTINE JENSEN DE LÓPEZ \\ Institute of Communication \& Psychology. University of Aalborg. Denmark \\ Kristine@hum.aau.dk
}

\begin{abstract}
This study explores the communicative production of gestural and vocal modalities by 8 normally developing children in two different cultures (Danish and Zapotec; Mexican indigenous) (16 to 20 months). We analyzed spontaneous production of gestures and words in children's transition to the two-word stage as well as interaction between children and their respective caretakers' use of gestural communication. Consistent with previous studies the results showed that all children used the gestural modality extensively across the two cultures. Two subgroups of children were identified regarding whether the children showed an early preference for the gestural or the vocal modality. Through Analyzes of two-element combinations of words and/or gestures, we observed a relative increase in cross-modal (gesture-word and two-word) combinations. The results are discussed in terms understanding gestures as a transition period and in relation to the degree to which gestures can be understood as a universal communicative device applied by children.
\end{abstract}

\section{Keywords}

Gesture, words, infancy, development, Danish, Zapotec, parent-infant interaction.

\section{Introduction}

Newborns seem to be equipped with primitive mechanisms for attuning into human communication and within their first year they gradually become more capable of acting on the physical world. However, they lack major communicative competencies. In the 70s Bruner set out the agenda to identify the non-linguistic devices that facilitate children in acquiring language (Bruner, 1975) defining the system in terms of the LASS (Language Acquisition Support System). Today gestures can be acknowledged as one of the centrals devices forming part of the LASS. This paper explores the transformation of infants' abilities to use sophisticated intentional actions, namely gestures, in the progress of learning to use words as their main communicative device. We also link the transformation of caregiver's use of child-directed gestures to the advance of the particular child in order to identify the dialectic context within which children learn to communicate conventionally.

The early hypothesis of Piaget (1962) and Werner and Kaplan (1963) stated that children's development of symbols originate from their interactions with objects. Although it seems intuitive for symbols to be vocal, e.g. expressed in words, it has seemed somehow less obvious that symbols also can be gestural, e.g. expressed in 
gestures. Early studies claimed that gestures function as an extension of the child's own body, however children's use of symbolic actions in the gestural modality has often been considered noncommunicative or nonreferential and has consequentially been recognized as "symbolic play" and hence analyzed separately from verbal actions. Words on the other hand have always been considered to be communicative or referential and remain to be the main focus in studies of early communicative development. The child's use of first words has been acknowledged as a central breakthrough in children's entrance into the conventional communicative system.

The early documentation of gestural schemes used productively by infants suggested that gestural schemes are used productively to communicate about a specific referent in a decontextualized, symbolic manner (Bates, Benigni, Bretherton, Camaioni \& Volterra, 1979). However this study only created minor interest back in the 70 s.

In the late 1970's language researchers began to explore children's acquisition of gestures now asking which role gesture plays in children's transition to early verbal communication. Although the early documentation of infants using gestural schemes productively to communicate about specific a referent in decontextualized, symbolic manner did not create large interest, researchers argued that there exists a continuity between prelinguistic and linguistic communication in which children are able to express communicative intentions gesturally prior to expressing them though conventional verbal symbols (Bruner, 1975; Bates, Camaioni, \& Volterra, 1975; Bates, Benigni, Brethorton, Camaioni \& Volterra, 1979: Volterra \& Erting, 1994/1990). For example the gesture of pointing was seen as an object-distinguishing tool and an important precursor of verbal object naming (Masur, 1990).

Since then questions have been raised on how to understand the relationship between gestural and verbal communication and the degree to which progress in one domain predicts progress in the other, and whether the relationship should be seen as a common mechanism or two independently developing mechanisms (Acredolo \& Goodwyn, 1990). It now seems a robust feature in the literature that infants tend to increase their gestural communication up to 18 months of age, followed by a decline, which seems to suggest that gesture as the main communicative device is replaced by conventional (verbal) communication (Lock, Young, Service and Chandler, 1990). Deaf children's development of sign language rather than verbal language shows a similar pattern in the initial communicative stages, although in their case gestural communication increases gradually developing into a complex system for gestural communication (i.e. the combination of two gesturally produced symbols (Caselli \& Volterra, 1990)), while hearing children break off into the verbal system for communication.

A longitudinal diary case study of an Italian infant reported that many of the gestures of the type "schemes of symbolic play" (holding the empty fist to the ear for TELEPHONE, waving the hand for BYE-BYE or raising the arms for TALL) were used frequently to communicate in a variety of contexts, and similar to those where first words are produced (Caselli, 1990). These types of gestures are now referred to as representational gestures and are contrasted to deictic gestures, such as pointing. It also seems that different types of gestures play different roles in this particular period of transition. The relationship between children's early gestural and verbal communication was later confirmed in a longitudinal study with 12 Italian-speaking children (Iverson, Capirci \& Caselli, 1994; Capirci, Iverson, Pizzuto and Volterra, 1996).

Infants' parallel development of spoken language and gesture is expressed through several phenomena; a) first gestures and first words emerge at around the same age (Caselli, 1990), b) children use gestures as well as words as initial communicative devices (Iverson, Capirci, \& Caselli, 1994) and c) children's achievements in gesture have shown to predict their progress in verbal language (Camaioni, Caselli, Longobardi, \& Volterra, 1991; Capirci, Iverson, Pizzuto, \& Volterra, 1996). Pioneer studies back in the 70s showed that onset of pointing is a reliable predictor of the appearance of first words (Bates, Benigni, Bretherton, Camaioni, \& Volterra, 1979), while attempts to explain the relationship between gesture and language 30 years later, showed that production of gesture-word 
combinations that convey two distinct pieces of information (e.g. the child points to the window while saying wow-wow as to express "there is a dog outside") predicts the emergence of two-word speech at the age of two years (Butcher \& Goldin-Meadow, 2000; Iverson \& GoldinMeadow, 2005).

Despite numerous studies showing that caregivers modify their speech in a consistent fashion when they interact with very young children $^{1}$ (Snow, 1995) only one study has addressed the notion of child-directed communication in the gestural domain longitudinally. In this study (Iverson, Capirci, Longobardi \& Caselli, 1999) they observed a group of Italian children at 16 and 20 months and found that at both age periods children's production of gesture was higher than that of mothers and maternal gesture production did not change significantly across the two age points. However, mother's gesture production was significantly related to the children's overall gesture production and there were large individual variations in the extent which mothers gesture to their children. Iverson et al. (ibid) did not examine whether individual mothers changed their childdirected gestures to match the developmental stage of the individual child in order to observer the mother's sensitivity to the individual development of their child.

The literature addressing what happens to the relationship between gesture and language after the transition to the two word stages is contradictory. Some argue that as spoken language takes over, children do not develop new gestures and that the frequency of gesture use reduces (e.g. Capirci et al. 1994), while recent research has shown that even school children produce gestures in spontaneous as well as complex cognitive settings (for example when explaining Piagetian conservation tasks (Alibali, Kita, \& Young, 2000).

Recently Stefanini, Caselli, \& Volterra (2007) have stated that the relationship between gesture and language in typically developing children is now well documented. However, based on comparisons of the development of hearing and non-hearing children Capirci and Volterra conclude that the particular communication system the child comes to use is shaped by the social interaction and the modalities used in social interaction (Capirci \& Volterra, 2008). In their conclusion they refer to social interaction at the macro level (hearing versus non-hearing societies), while disregarding social interaction at the micro level (middle-class versus non middle-class, high-gestural versus low-gestural societies). The motivation of the present study is to explore the extend to which gesture can be seen as a transitional device en route to twoword speech in a cross-cultural micro level perspective. In particular we set out to contribute with data that can support the recent claim stated by Stefanini, Caselli and Volterra (2007) that the relationship between gesture and language is a robust transition period in development. If the particular stage of transition from communicative gestures to words is to be accepted as a universal period of transition expressed in the development of all children independent of their culture and language, it lacks support from the communicative development of children living in non-rich gestural cultures and non middleclass societies.

Placed on a continuum of high and low gesturing societies, it is our experience that Italy is best categorized as a high gesturing society compared to Denmark (and Scandinavian), which is seen as a low gesturing society (Kendon, 1992). By investigating the transition from gesture to word in a group of Danish children we are able to explore the existence of a robust relationship between gesture and spoken language as parallel systems in the initial stages of communicative development. Furthermore, we explore this same relationship in non-western and non-middle class children through observations of Zapotec children's gestural and vocal actions (Zapotec is a Mexican indigenous society and language). Extensive periods of ethnographic fieldwork in the Zapotec society suggest that this particular Zapotec society is a low gestural culture.

The research question we ask is whether Danish and Zapotec infants employ gestures and

\footnotetext{
1 This interaction is known as "motherese" or "child-directed speech".
} 
gesture-word combinations as a robust feature of communicative development, which precedes the two-word stage. We employ the notion of robustness in relation to the degree that our longitudinal results from Danish and Zapotec children support the results from the Italian study of 12 children reported by Iverson, Capirci, \& Caselli, (1994) and Capirci, Iverson, Pizzuto, \& Volterra, (1996). In addition we compare the children's gestures with the caregivers' child-directed gesture to examine changes in the input gestures as a function of the child's development.

\section{Methods}

\subsection{Participants}

A total of 8 children participated in the study. Six children were Danish children ( 3 boys and 3 girls) from middle-class families and two children (boys) were Zapotec children living in an extremely low-income remote rural community in the Southern State of Oaxaca (Jensen de López, 2006 for a description of the culture). All children were monolingual native speakers of the respective languages. Four of the Danish children were selected through personal communication and the remaining two children form part of the Plunkett corpus registered in CHILDES database (Plunkett, 1993). The Zapotec children were selected from a larger group of children observed as part of a longitudinal study of Zapotec language acquisition and socialization (Jensen de López, 2002).

\subsection{Procedure}

Two observations were conducted for each child: one at 16 months and the other at 20 months of age. Each session was videotaped and lasted 45-60 minutes, during which caretakers were instructed to interact and play with their child as they normally would. The recordings contained two different scenarios: play with objects and meal or snack time. The first 40 minutes starting 5 minutes into the recording were transcribed and analyzed. A total of 30 minutes of the recorded data, starting 5 minutes into the recording was transcribed and coded in two modals; first in the gestural modal and then in the vocal modal.

\subsection{Coding}

The coding procedure was in accordance with the methodology described in the Capirci, Iverson, Pizzuto and Volterra (1996) study. All communicative and intelligible gestures and words were transcribed from the videotapes. Gestures and words were considered to be communicative if they were accompanied by eye contact with another person, vocalization, or other clear evidence of an effort to direct the attention of another person present in the room (Thal \& Tobias, 1992). Only communicative acts with identifiable referents were considered in the analysis. We employed a broad definition of what constituted a gesture and a word because children's first gestures and words can be idiosyncratic and constructed in the child-caregiver context. The Zapotec transcriptions were carried out in consultation with a native Zapotec speaker.

\subsubsection{Gestural Production}

All intentional gestures produced by the child and directed towards the parent (caregiver) were coded in accordance with the coding procedure developed at Italian National (CNR) Institute of Psychology in Rome ${ }^{2}$. All gestures were classified as deictic or representational. Deictic gestures were gestures that refer to an object or an event by indicating the referent. The form of the deictic gesture does not directly resemble its referent, thus the meaning of these gestures can only be determined through reference to the context in which the gesture is communicated. We coded four types of deictic gestures: Giving, Showing, Pointing and Requesting such as reaching. An example of the gestures coded as a Giving gesture is if the child held an object towards an adult. Gestures were classified as a Showing gesture if the child held up an object in the adult's line of sight. A gesture was

\footnotetext{
${ }^{2}$ Now the Institute of Cognitive Sciences and Technologies.
} 
coded as Pointing if there was clear evidence of extension of the index finger towards a specific object or event. In accordance with Thal and Tobias (1992) tapping a location or object with the hand were also coded as pointing gestures. Finally, Requesting gestures consisted of an extension of the child's arm in the direction of an object or event, sometimes with repeated opening and closing the hand.

Representational gestures differ from deictic gestures by representing specific referents, and in that their semantic content does not change with the context. Gestures coded in this category referred to an object, person, location, or event and were expressed through hand movement, body movement, or facial expression. Three subcategories of representational gestures consisted of Action, Social and Culturally accepted gestures. Examples of representational gestures are shaking the head for NO, waving for BYE-BYE, raising the arms for pick-me-up or copying the movement performed by the object (e.g. turning the arms in circular movements for BICYLE or flapping the hands for BIRD).

\subsubsection{Verbal Production}

All words were transcribed from the videorecordings ${ }^{3}$. All the Zapotec recordings were transcribed and checked across the video data by native Zapotec speakers who were the main caregivers of the children.

\section{Word Types:}

Words were classified as deictic or representational according to the same criteria used for gestures. Deictic words included pronouns, adjectives and locatives (e.g. I, there). All other words (e.g. nouns, proper names verbs, adverbs, functional words and closed-class words) were coded as representational words. However, in defining these categories, we are not assuming that infants posses mental representations identical to the lexical organization of adults.

\subsubsection{Intercoder reliability}

The first 10 minutes of each datapoint was coded by two independent coders for the codings of deictic and representational gestures and for identification of a gesture. Results showed $89 \%$ and $91 \%$ of agreement respectively.

\section{Results}

Extensive communication in both the gestural and the vocal modalities was observed in all subjects and across the two groups of children. At both 16 and 20 months of age, the Danish and the Zapotec children produced single element vocabularies in the verbal and gestural modalities and combinations of two or more elements within and across modalities. As expected from the results from the Italian study we observed a substantial amount of variability among individual children.

I start by describing the data from the 16month observations followed by analyses of the diversities and semantic content of gestural and vocal vocabularies. We then proceed to describe the observations of the children at 20 months of age following the same structure. Due to the relative small Zapotec sample, the results from this group only form part of the comparison when appropriate.

\subsection{6-Month-Olds}

\section{Analysis of gestural and verbal production}

In order to highlight individual differences in the children's gesture and word production we present the data reflecting the patterns exhibited by the children. Figure 1 shows the total number of gestures and words, independent of types, produced by each child at the age of 16 months ${ }^{4}$. The data produced by the Zapotec children can be identified by the suffix $-Z$ e.g. EstebZ. Similar to the results from the Italian

3 Transcriptions for words were available in CHILDES for the Plunkett data, however due to minor disagreements between these transcriptions and those of the coding group, we checked all transcriptions and recoded where necessary.

4 All names are anonymous. 
Figure 1. Number of words and gestures produced by each child at 16 months.

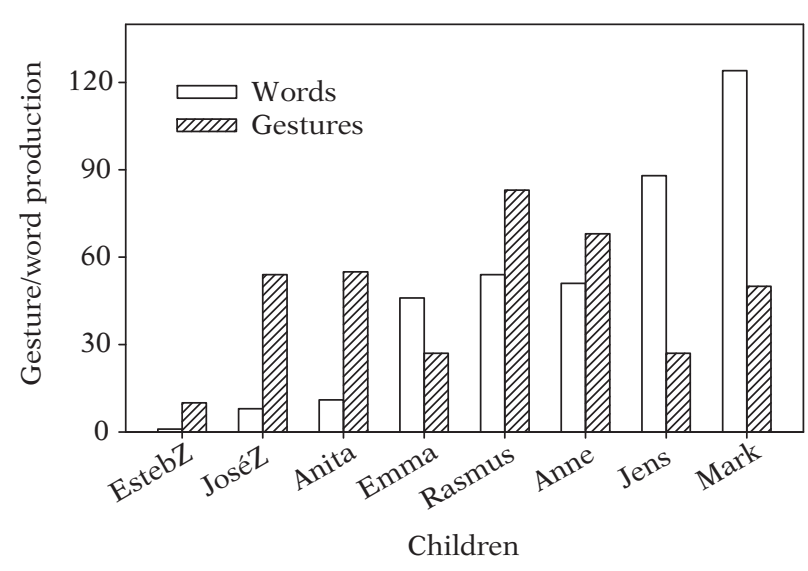

children (Iverson, Capirci \& Caselli, 1994) we identified two subgroups within the Danish group of children.

Three Danish children (Anita, Rasmus and Anne) produced a higher proportion of gestural than verbal vocabularies, while the remaining three children produced more words than gestures. Both Zapotec children produced more gestures than words, despite an overall production of one of the Zapotec children (EstebZ) being extremely low. Compared to the production of the Italian children, the frequency of gestures (measured in tokens) produced by the Danish children was in general higher as was the range within the repertoires of the children (27-83 for the Danish children and 5-14 for the Italian children). Consistent with the results for the Italian children the variability in the Danish children's production of words was much more apparent than the variability in the production of gestures (11-124 compared to 1-73) and as for the gesture production more frequent. The difference between the children's production of gestures compared to words was non-significant on a paired-samples t-test at $(M=52, S D=22.23$ for gesture) to age 16 months $(M=62, S D=$ 39.88 for words), $t(5)=-.53, p=.68$.

The overall pattern for the children across the two cultures show that they displayed a preference for communication in either the gestural or the verbal modality with a higher proportion of children (5 of 8 ) demonstrating a preference for gestural communication at the age of 16 months. Only two of the children, where both had very large verbal vocabularies (Jens and Mark), produced extensively more words than gestures, yet they still showed preference for communication in the gestural modality. When excluding these two children the production of gestures clearly exceeded production of words at 16 months $(M=$ $50, S D=26.67$ for gesture and $M=29, S D=24.27$ for words), with the differences approaching a significant level $t(5)=2.10, p=.089$.

Analysis of the different categories of gestures produced by the children showed that all the children with the exception of one (Jens) showed a robust pattern producing a higher proportion of deictic $(M=38, S D=20.28)$ than representational gestures $(M=20, S D=16.90)$. This result is similar to what was observed in the Italian study (Iverson, Capirci \& Caselli, 1994), although their results, with a relatively larger sample, showed a significant difference, while the difference found in our relatively small sample was non-significant. Within the deictic categories POINTING accounted for a substantial proportion of the total deictic gestures produced and was employed more often than SHOWING, REQUESTING or GIVING (see Figure $2 \mathrm{a}$ and Figure $2 \mathrm{~b}$ ).

This pattern was consistent at the age of 16 and 20 months and consistent across the Danish and Zapotec groups. The results for the Italian children showed a robust pattern of preference for POINTING as opposed to the remaining categories of deictic gestures for each individual

\section{Figure 2a. Distribution of number of gesture types produced by Danish children at age 16 and 20 months.}

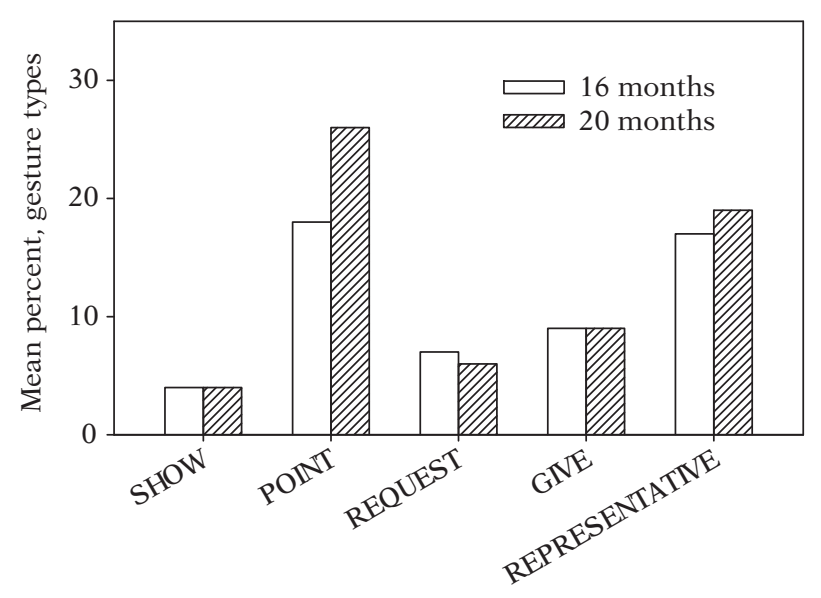




\section{Figure 2b. Distribution of number of gesture types produced by Zapotec children at age 16 and 20 months.}

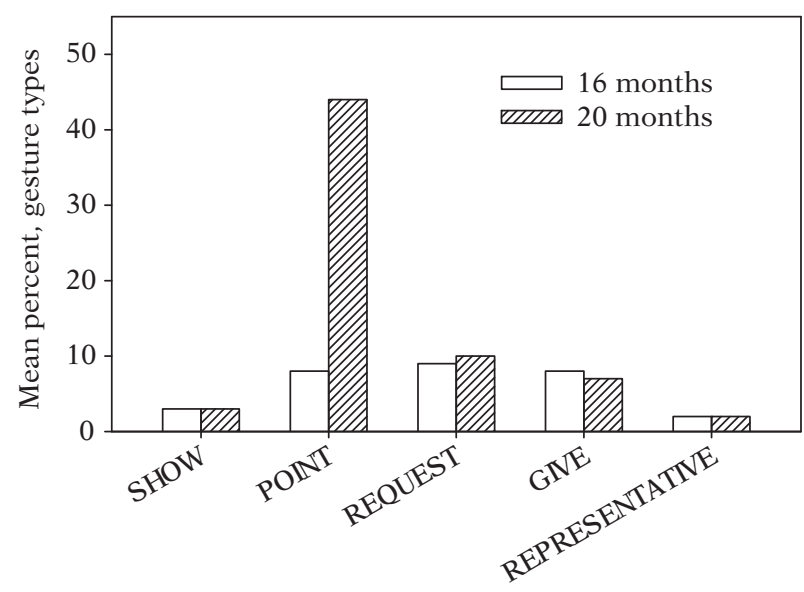

child. However, we were not able to identify this robust pattern for each individual child in our study. Half of the children (Anne, Emma, Rasmus and Mark) produced more POINTING gestures than the remaining categories of deictic gestures, one child (JoséZ) produced an equal proportion, while the remaining three children (EstebZ, Anita and Jens) produced slightly less POINTING gestures compared to the remaining categories of deictic gestures. Recall that the results from the analysis of gestures and words showed that Anita and Jens fall into two distinct subgroups; hence their shared preference for gesture types cannot be linked directly to their preference for gestures versus words.

\subsection{0-Month-Olds}

\section{Analysis of gestural and verbal production}

The number of different word and gesture types (both deictic and representational) produced by individual children at 20 months is illustrated in Figure 3. As illustrated in figure 3, the composition of the two subgroups observed at 16 months transformed dramatically at 20 months of age. A significantly larger proportion of children had more communicative elements in the vocal than in the gestural modality (Wilcoxon $z=-2.4, p=02$ ). This pattern is consistent with the pattern observed in the Italian study (Iverson, Capirci \& Caselli, 1994).

\section{Figure 3 Number of words and gestures produced by each child at 20 months.}

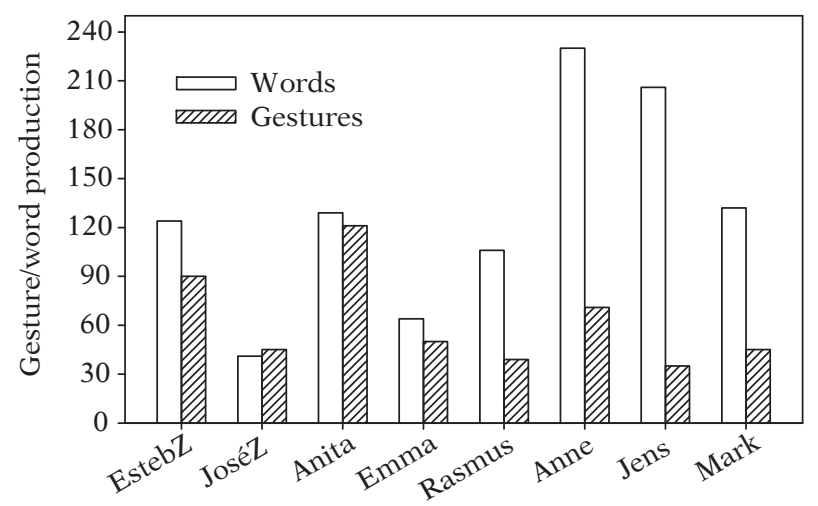

Of the eight children, only one child (JoséZ) used an equal amount of gestures and words in his communication. A high degree of individual variability with respect to production of both words (range 64-230 for Danish children) and gestures (range 35-121 for Danish children) remained evident at 20 months. The Danish children maintained a higher production of words compared to the Italian children. When comparing individual patterns of production at 16 and 20 months, a substantial increase in the overall number of communicative (verbal or gestural) acts and production of words was observed in all children (Wilcoxon $z=-2.5$, $p=.012)$. Although the use of gesture increased in most children (5 out of 8 children) at 20 months this difference, as in the case of the Italian children, was not statistically significant. However, when controlling for the three children that decreased their gesture production (Rasmus, Mark and JoséZ) the increase in the gesture for the remaining five children reached a significant level (Wilcoxon $z=-2.02, p=.043$ ). Hence, again this may reflect two subgroups of children on different stages of their language development. It is important to point out that although the overall level of gesture production increased from 16 to 20 months, there was a proportionate decline in the production of gesture relative to words at 20 months. Gesture accounted for $49 \%$ and $33 \%$ of the child's total communication (gestural and verbal) at 16 and 20 months, respectively. Contrastive to this finding, the proportion of communication consisting of speech increased substantially, from 50\% at 16 months to $67 \%$ at 20 months. The increase 
in the groups production of words was significant $(M=48, S D=42.43$ for age 16 months and $M=129, S D=62.98$ for 20 months) $Z=-2.52$, $p=.012$. Again this pattern is similar to the pattern observed in the Italian study (Iverson, Capirci \& Caselli, ibid).

The Italian study reported a significant increase in the production of deictic gestures and a significant decline of representative gestures from 16 to 20 months. We also observed the developmental pattern of deictic gestures increasing $(M=36$ to $M=51)$ and representative gesture decreasing slightly $(M=14$ to $M=12)$ from 16 to 20 months, however none of these differences were significant (see figures $2 \mathrm{a}$ and $2 \mathrm{~b}$ ). We speculated that the relatively low production of representational gestures produced by the Zapotec children may explain this and carried out new Wilcoxon Tests for the Danish group only. The differences for the Danish children however, remained non-significant. When investigating the individual profiles for each of the children we observed a subgroup of three children (Anita, Emma and Rasmus) who increased their production of representative gestures from 16 to 20 months and oppositely a subgroup of three children (Mark, José and Rasmus) who decreased their production of deictic gestures from 16 to 20 months $^{5}$. It is noteworthy that Anita and Mark both already produced a high level of verbal communication at 16 months. Because deictic gestures accounted for a substantial number of the gestures produced at both age periods, we explored the patterns of usage of the four types of deictic gestures. The distribution of the different deictic gesture types is presented in Figures $2 \mathrm{a}$ and $2 \mathrm{~b}$ above.

The deictic gesture types SHOWING, REQUESTING and GIVING were all used regularly by both groups of children and at both age periods, and although they accounted for a relatively low proportion of the total deictic gestures, the usage was stable at across both age periods. This result contrasts with the pattern for the Italian children, which showed a non-significant decline at 20 months $^{6}$. The most frequently utilized deictic gesture POINTING at both age periods was also the only type of ges- ture that increased at 20 months. However, the increase was not significant. This is consistent with the pattern produced by Italian children, which showed a significant increase across development for pointing.

\subsection{Analysis of cross-modal communication}

In the remaining analysis we focus on the structure and content of the children's gestural and vocal utterances comparing our results with the results from the Italian children analyzed and reported in the Capirci, Iverson, Pizzuto and Volterra (1996) study. Hence we ask how infants use gestures, words and the combination of gestures and words in their early communication. Figures $4 \mathrm{a}$ and $4 \mathrm{~b}$ summarize the general patterns in the two cultural groups production of gestural, vocal and cross-modal utterances at 16 and 20 months.

The figure shows the mean number of all occurrences (tokens) of single-, two- and multielement gestural/and or vocal utterances for the two groups at 16 and 20 months. At 16 months children's communication in both groups consisted primarily of single words and single gestures, but crossmodal combinations (gestureword) of two elements were relatively common.

\section{Figure 4a. Mean number of tokens of different utterances produced by the two groups of children at 16 months.}

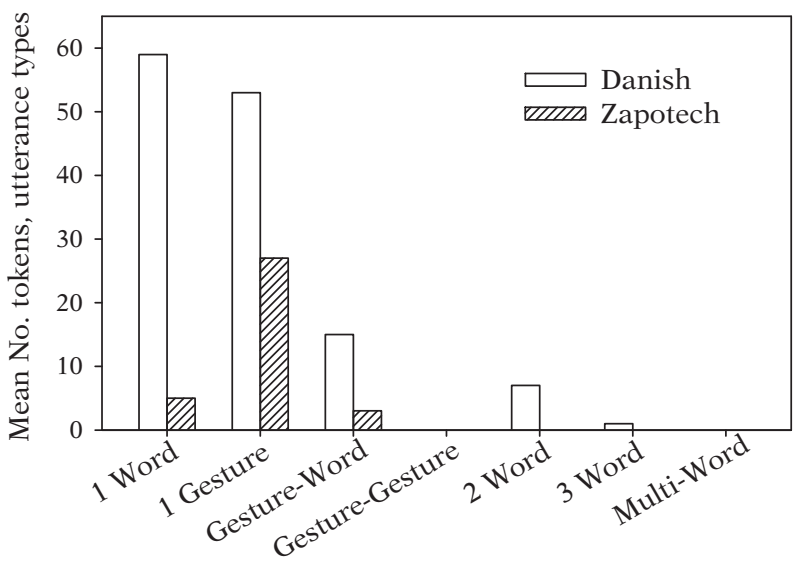

\footnotetext{
5 The standard variations also suggest large individual differences.

6 The Italian study did not code the deictic gesture type GIVING, which we coded in our study.
} 
Figure 4b. Mean number of tokens of different utterances produced by the two groups of children at $\mathbf{2 0}$ months.

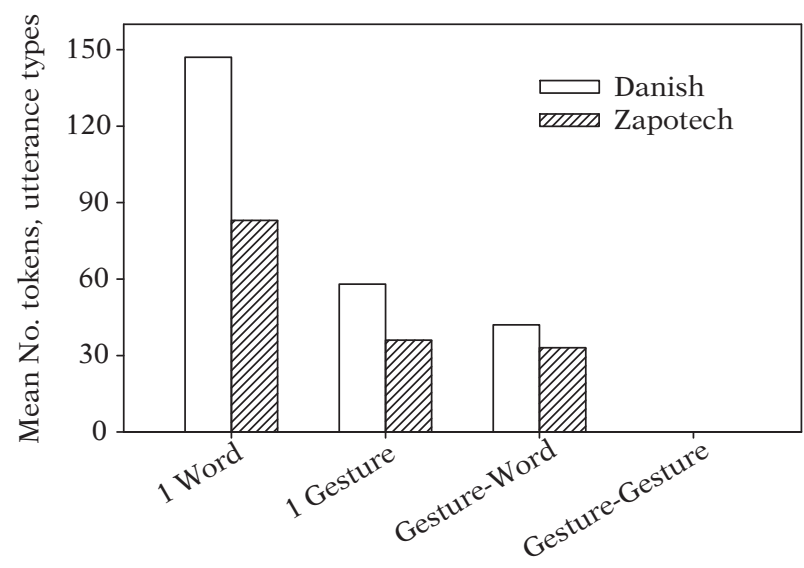

In contrast there was a very small number of unimodal combinations (multi-words and multielement) and no productions of two gesture combinations. Overall the production of the Danish children was higher than production of the Zapotec children, although the general pattern was the same. By the age of 20 months the number of single-word and multi-element (gesture-word) combinations had more than doubled (from 64 to 230 and from 18 to 75 ), whereas the increase in the single gesture productions was low (from 80 to 94 ). None of the children produced combinations of two-gesture utterances at any of the age periods. This pattern is consistent with the pattern of the Italian children ${ }^{7}$.

The following analysis will focus on the modality of production, structure, and information content of the two-element combinations. Codings of all crossmodal combinations produced by the children were classified according to the three types of combinations (or classes) described and identified in the Capirci et al. (1996) study. Information conveyed by EQUIVALENT (=) combinations consisted of combinations were the gesture and word referred to the same referent. COMPLEMENTARY (\&) combinations consisted of a gesture and word referring the different features of the same referent, while SUPPLEMENTARY (+) combinations were identified as gesture-word combinations, with the same or different referent, BUT with each element adding adding information to the other (e.i. the child points to a doll and says SLEEP). The results are illustrated in figures 5a and $5 \mathrm{~b}$. The number of SUPPLEMENTARY combinations was smaller relative to the other two combination types for the Danish children at both age periods.

Figure 5a. Number of Equivalernt, Complementary, and Supplementary twoelement combinations produced by Danish children at age 16 and 20 months.

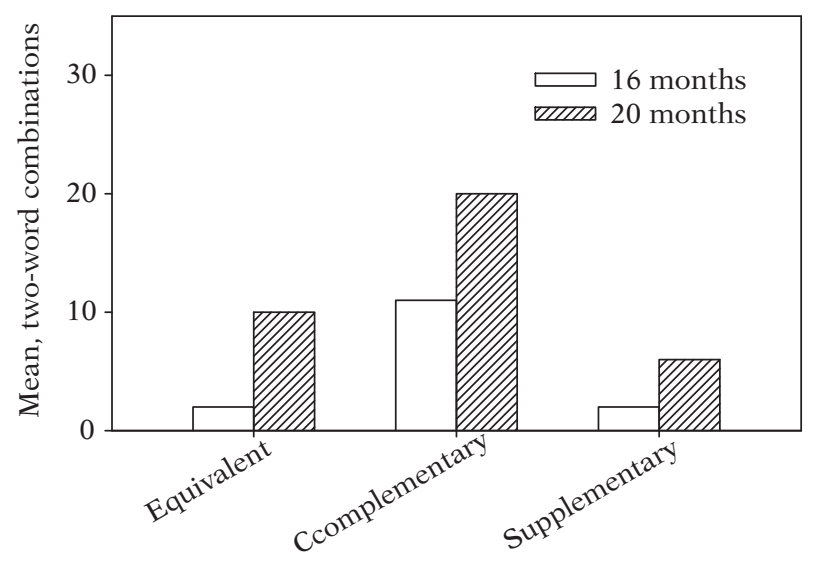

5b. Number of Equivalernt, Complementary, and Supplementary twoelement combinations produced by Zapotec children at age 16 and 20 months.

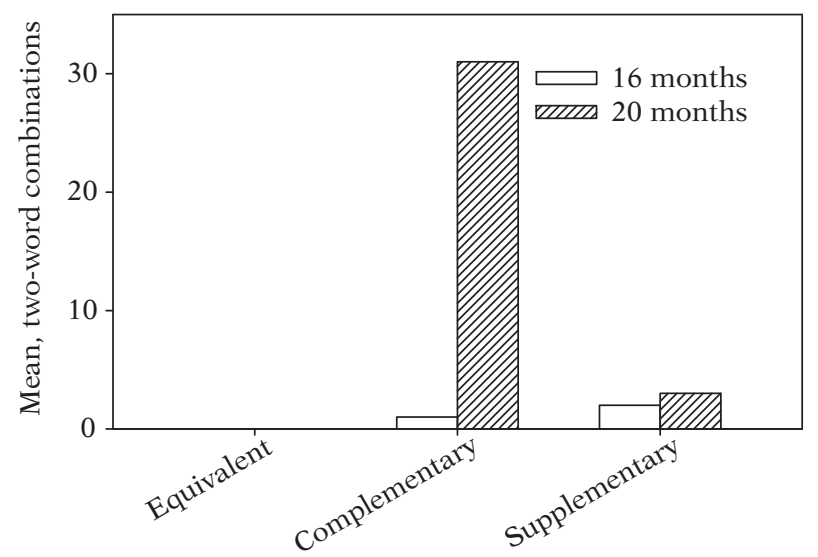

7 The combination of two-gesture utterances is documented in the communication of deaf children, but not in hearing children (see Volterra \& Erting, 1994/1990). 
When looking at the developmental changes in production of different combination subtypes, within the COMPLEMENTARY class, crossmodal combinations of a deictic gesture and a representational word (DG\&rw) (i.e. the child points to her food and says mad (food)) and the combinations of a deictic gesture and a deictic word (DG\&dw) (i.e. the child points to a cup and says det (that)) were the most frequently produced at both ages and across the two cultural groups. The Zapotec children produced these combination classes less frequent when compared to the Danish children at 16 months, but then increased substantially at 20 months.

Within the EQUIVALENT class all combinations consisted of a representational gesture and a representational word $(\mathrm{RG}=\mathrm{rw})$ (i.e. child shakes his head and says nej (no), but this combination class was only produced by Danish children. Within the SUPPLEMENTARY class we observed a very small number of productions, across both cultural groups. This contrasts with the pattern of the Italian children, who showed a clear increase in the development of supplementary combinations from age 16 to 20 months.

We then asked whether gestures and gesture-word combinations predict subsequent lin- guistic development? We have showed patterns that suggests the existence of a developmental relationship between gesture-word combinations and two word utterances. In Table 1 we present the presence $(+)$ and absence $(-)$ of the three classes of gesture-word combinations and of two-word utterances for each child's production at age 16 and 20 for the two cultural groups.

The table shows that two of the children (Anita and Emma) produced few or no gestureword combinations parallel to not producing two-word utterances at 16 months. At 20 months most of the Danish children were producing all three classes of combinations, including Emma, although she was still not producing two-word utterances. This pattern shows a relationship, which can be said to be less robust, compared to the relationship observed in the Italian study (Capirci et al.) where seven of the twelve children produced gesture-word combinations at 16 months before producing twoword utterances. Hence, Danish children might be seen as developing two-word utterances at an earlier age than Italian children. In any case data from all three cultures suggest a gradual development of gesture-word combinations parallel to the development of two-word utterances.

Table 1. Presence (+) or absence (-) of amodal combinations and two-word utterances in Danish children's production at 16 and 20 months.

\begin{tabular}{ccccc}
\hline \multicolumn{5}{c}{ GESTURE-WORD } \\
\hline Child & Equivalent & Complementary & Supplementary & Two-word \\
& $\mathbf{1 6}$ Months (N = 6) & . & - \\
Anita & - & - & - & - \\
Emma & + & + & - & + \\
Rasmus & + & + & + & + \\
Jens & - & + & + & + \\
Anne & + & + & + & + \\
Mark & + & Complementary & Supplementary & Two-word \\
Child & Equivalent & $\mathbf{2 0}$ Months (N = 6) & + \\
& + & + & - & + \\
Emma & + & + & + & + \\
Jens & + & + & + & + \\
Anita & + & + & + & + \\
Rasmus & + & + & + \\
Anne & + & + & + \\
Mark & & + & + \\
\hline
\end{tabular}


Our final question was whether input factors play a role in infants' gestural and verbal communication. If one expects verbal communication to be the main facilitating input factor scaffolding the infant in his transition from gestural communication to verbal communication, we should expect caregivers to produce few gestures when communicating with their infants. We coded all child-directed (caregiver-directed) gestures employing the same coding schema we had applied in accounting for the children's gestural communication at 16 and 20 months. The results were then matched with the gestural production of each individual child. Figures $6 \mathrm{a}$ and $6 \mathrm{~b}$ illustrate the relationship between the caregiver and the infants' gestures at 16 and 20 months.

Figure 6a. Gesture production by caregivers and individual children at 16 months.

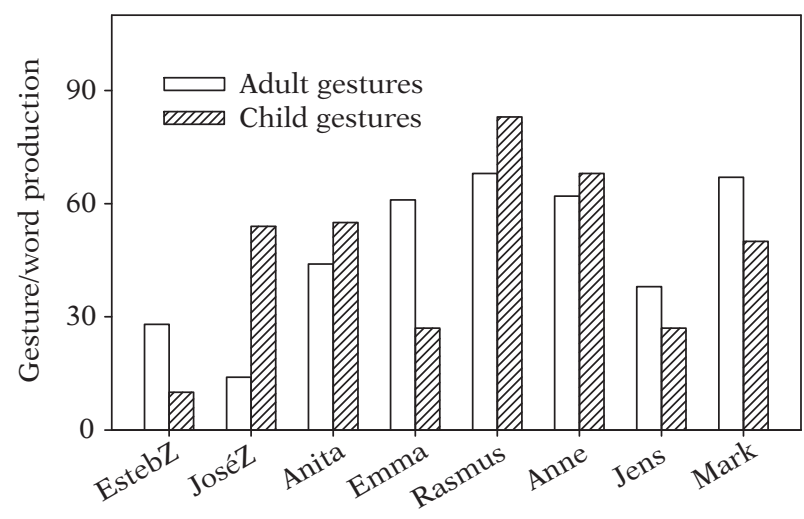

Figure $6 \mathrm{~b}$. Gesture production by caregivers and individual children at $\mathbf{2 0}$ months.

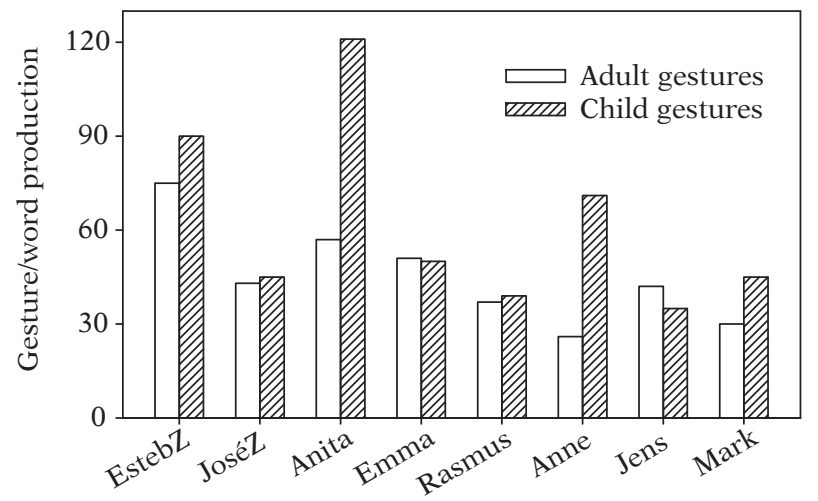

At 16 months the caregivers of four of the children (JoséZ, Anita, Emma and Jens) produced more gestures than their respective infants, whereas the remaining four caregivers produced less gestures than their respective infants. By the age of 20 months this discrepancy had either disappeared or was reversed. In four of the caregiver-child dyads production of gestures was equal (JoséZ, Emma, Rasmus and Jens). Three of these dyads were the same dyads were caregivers produced a higher frequency of child-directed gestures compared to the child at 16 months. In the remaining dyads the child was the predominant producer of gestural communication. This change in the pattern of "who gestures to whom" seems to be mediated by the child's gradual entrance into the verbal modality. Once the child shows competence in the verbal modality caregivers intuitively decrease their gestural communication directed towards the child as a scaffolding of the child within conventional linguistic communication. It is important to note that caregivers decrease, BUT not avoid altogether gestural communication with their respective children, once the child shows the first signs of verbal competence. This suggests that gestural communication continues to play a role, although it has different functions throughout development even for adults that have acquired the conventional linguistic system of communication.

\section{Discussion}

The purpose of the present study was to contribute to our understanding of infants early communicative development through the investigation of the role that gesture plays during the transition to two-word speech by children living in two very different low gesturing cultures. We raised the question of whether the transition period identified in Italian children's communicative development is a robust and universal stage and of what role parental input plays in children's production of gestures and words. We addressed these questions by examining spontaneous gestural and vocal communication in a longitudinal study of six Danish and two Zapotec children at two age points. In our view the results obtained from a relatively small number of children support several of the initial 
findings from Italian children, while questioning the robustness of the transitional period from gesture to two-word utterances. Furthermore, we contribute with original knowledge of the interaction between parental input in the gestural modality and infants' early communicative development.

First, when addressing the issue of the advantage of gestural communication and gestureword communication en route to two-word utterances, we observed strong individual differences and large variability across the children with respect to whether gesture was the only communicative device at 16 months as showed by Italian children (Capirci et al. 1996). This difference was expected from similar observations in the studies of Italian children, and suggests a robustness of the existence of separate subgroups of children at this age. The overall frequency of gestures produced by the Danish children was higher than observed in the Italian group, which suggests that Italy which is considered a high gesturing culture does not enhance children's frequency of gestural production. Due to the individual differences we did not find a clear preference for communication in the gestural modality at 16 months, nor a significant difference between communications of gestures versus words. This may be due to our sample size. However, we were able to stress the fact that gesture is an important communicative device for infants and that representational gestures have a communicative function rather than merely serving as symbolic play. Vygotsky argued that in order to detach the meaning from an object children need to find a pivot in something else (Vygotsky, 1978). Gestures can in this sense be seen as pivots for meaning and as properties of things in a similar way as word can be seen as pivots for objects.

The Danish and Zapotec children showed a robust preference pattern for producing deictic gestures compared to representational gestures as well as a preference for pointing gestures as opposed to the gesture types showing, requesting and giving. This pattern is similar to the pattern produced by Italian children and suggests a universal robust preference for deictic gestures, in particular pointing gestures, across children from very different cultural contexts. The pattern of the remaining deictic gestures does not reflect cross-cultural similarities. Hence, these types of gestures may be more sensitive to the specific cultural context than pointing gestures. However, more longitudinal and cross-cultural information is required to confirm this difference.

The developmental change at 20 months also reflected some of the patterns observed for Italian children. First, a robust transition from gestures to words was observed in that most children produced more vocal compared to gestural communication. There was a significant pattern of increase in the children's overall communicative acts and words. Oppositely the increase in the gestural modality was minor and not significant. However we found individual differences suggesting two subgroups: those children who increased their frequency of gestural productions at this age and those who showed a clear decrease. We do not interpret this result to be in conflict with the overall argument that gestures may serve as a vehicle for children in their development into becoming conventional language users. Rather we view the emergence of subgroups to reflect same age children on different platforms of their language development.

We did not observe a decrease in the children's production of deictic gestures at 20 months and the distribution of the types of deictic gestures showed a slight different pattern than that observed in the Italiean study. The cross-modal observations revealed an expected striking increase in gesture-word combinations, while none of the children produced gesturegesture combinations suggesting that gestural and cross-modal communication function to enrich the child's communicative device at a stage where its conventional linguistic abilities yet is in an emergent stage. The information conveyed in the children's productions of two-element combinations expressed the usage of the three classes of combinations, namely complementary, equivalent and supplementary similar to what is observed in the production of the Italian children, however the overall pattern differed. We were not able to identify a clear prediction of gesture and gesture-word combinations in relation to the children's transition to the twoword stage, although it does seem that all children proceed through gesture and gesture combinations en route to their development of two-word and multi-word utterances. 
Finally, we observed an original result that should be taken into account when investigating children's early communicative development, namely the tight relationship between caregivers child-directed gestures and the child's production of gestures. It seems that parents initially anchor their communication in the gestural modality and as the child advances in his linguistic competences, calibrates the gestural device. Hence our data shows a high level of parental sensibility towards the individual child's zone of proximal development in the communicative domain and gradually makes use of more decontextualized communication gradually directing the child towards becoming a proficient communicative participant. This underlines the role of social interaction in early communicative development (Vygotsky, 1978; Bruner, 1983). Our results contribute to the results of child-mother gestural communication reported by Iverson et al. (1999) from a level og micro analysis, which makes it possible to explore mother-child interaction in a closer detail. Although our results overall contradict the results from the Italian study, when taking into account the different levels of analysis in the two studies our results simply stress the notion of individual differences in gestural communication similar to what is documented for early verbal communication. Both studies support the fact that parents do not suddenly drop their gestural communication to their children once the child has entered the two-word phase, but maintain to communicate with the child in the gestural modality, while adjusting it to meet the competences and zone of proximal development of the child. Hence, gestures, although from an evolutionary perspective might have emerged as a mere communicative device, do not serve only to communicate. They have changed their function to serve as a supportive system parallel to language (McNeill, 2005). With Capirci and Volterra, we view a reappraisal of the definition of the one-word and two-word stage of communicative development (Capirci \& Volterra, 2008).

The established observation of a tight relationship between gesture and words has clear implications for intervention with clinical groups of children with communicative problems for example children with Down's syndrome, for identifying children at risk of lan- guage delay as well as for understanding the evolution of language (see Stefanini et al., 2007).

\section{Acknowledgements}

The work reported in this paper was partially supported by European Science Foundation EUROCORES Programme OMLL and the NASUD project through the Danish Research Council of Culture and Communication. Parts of this paper were presented in a symposium titled: "A cross-linguistic and cross-cultural study of the role of gesture in early development" at the International Association for the Study of Child Language Congress, July 25th, Berlin, Germany. We thank Lena Lippke and Ane Knüppel for their assistance with the coding. A similar version of this study is published in Danish in Psyko \& Logos, 2. 29. Dansk Psykologisk Forlag, 2008.

\section{References}

Acredolo, L. P. \& Goodwyn, W. (1990): Sign language among hearing infants: the spontaneous development of symbolic gestures. In Virginia Volterra \& Carol J. Erting (Eds.), From gesture to language in hearing and deaf children. Berlin: Springer-Verlag. (1994-2 ${ }^{\text {nd }}$ Edition, Washington, DC: Gallaudet University Press).

Bruner, J. S. (1974). From communication to language: A psychological perspective. Cognition, 3(3), 255-287.

Alibali, M. W., Kita, S. \& Young, A. J. (2000): Gesture and the process of speech production: we think, therefore we gesture. Language and Cognitive Processes, 15, 593-613.

Bates, E., Benigni, L., Bretherton, I., Camaioni, L. \& Volterra, V. (1979): The emergence of symbols: cognition and communication in infancy. New York: Academic.

Bates, E., Camaioni, L. \& Volterra, V. (1975): The acquisition of performatives prior to speech. MerrillPalmer Quarterly 21, 205-26.

Bruner, J. (1975). From communication to language: a psychological perspective. Cognition, 3, 255287. 
Bruner, J. (1983). Child's Talk. New York: Norton.

Butcher, C. M. \& Goldon-Meadow, S. (2000): Gesture and transition from one- to two-word speech: when hand and mouth come together. In D. McNeill (Ed.). Language and Gesture (pp. 235-258). Cambridge: Cambridge University Press.

Camaioni, L., Caselli, M. C., Longobardi, E. \& Volterra, V. (1991): A parent report instrument for early language assessment. First Language, 11, 345-59.

Capirci, O. \& Volterra, V. (2008): Gesture and Speech: the emergence and development of a strong and changing partnership. Gesture 8:1, 22-4.

Capirci, O., Iverson, J. , Pizzuto, E. \& Volterra, V. (1996): Gestures and words during the transition to two-word speech. Journal of Child Language, 23, pp. 645-673.

Caselli, M. C. (1990). Communicative gestures and first words. In Virginia Volterra \& Carol J. Erting (Eds.), From gesture to language in hearing and deaf children. Berlin: Springer-Verlag. (1994-2 ${ }^{\text {nd }}$ Edition, Washington, DC: Gallaudet University Press).

Caselli, M. C. \& Volterra, V. (1990): From communication to language in hearing and deaf children. In Virginia Volterra \& Carol J. Erting (eds.), From gesture to language in hearing and deaf children. Berlin: Springer-Verlag. (1994-2 ${ }^{\text {nd }}$ Edition, Washington, DC: Gallaudet University Press).

Iverson, J., Capirci, O. \& Caselli, M. C. (1994): From communication to language in two modalities. Cognitive Development, 9, pp. 23-43.

Iverson, J., Capirci, O., Longobardi, E. \& Caselli, M. C. (1999): Gesturing in mother-child interactions. Cognitive Development, 14, 57-75.

Jensen de López, K. 2006. Culture, Language and Canonicality: Differences in the Use of Containers between Zapotec (Mexican indigenous) and Danish Children. In A. Costall \& O. Dreier (eds.) "Doing things with Things: The Design and Use of Everyday Objects" London: Ashgate.

Jensen de López, K. (2002): Baskets and Body-Parts: A Cross-Cultural and Cross-Linguistic Investigation of Children's Development of Spatial Cognition and Language. Ph.D. dissertation. Department of Psychology, Aarhus Universitet, Denmark.

Kendon, A. (1995): Some recent work from Italy on quotable gestures (emblems). Journal of Linguistic Anthropology, 2, 92-108.

Lock, A., Young, A., Service, V. \& Chandler, P. (1990): Some observations of the origin of pointing gesture. In Virginia Volterra \& Carol J. Erting (eds.), From gesture to language in hearing and deaf children. Berlin: Springer-Verlag. (1994-2 ${ }^{\text {nd }}$ Edition, Washington, DC: Gallaudet University Press).

MacNeill, D. (2005): Gestures and thought. Chicago: University of Chicago Press.

Mazur, E. F. (1990): Gestural development, dual-directional, signaling, and the transition to words. In Virginia Volterra \& Carol J. Erting (eds.), From gesture to language in hearing and deaf children. Berlin: Springer-Verlag. (1994-2 ${ }^{\text {nd }}$ Edition, Washington, DC: Gallaudet University Press).

Piaget, J. (1962): Play, dreams and imitation in childhood. New York: Norton.

Plunkett, K. (1993): Lexical segmentation and vocabulary growth in early language acquisition. Journal of Child Language, 20, 43-60.

Stefanini, S., Caselli, M. C. \& Volterra, V. (2007): Spoken and gestural production in a naming task by young children with Down syndrome. Brain and Language, 101 (3), 208-221.

Thal, D. \& Tobias, S. (1992): Communicative gestures in children with delayed onset of oral expressive vocabulary. Journal of Speech and Hearing Research, 35, pp. 1281-9.

Vygotsky, L. V. (1978): Mind in society: the development of higher psychological processes. Edited by Cole, M.; John-Steiner, V., Scribner, S. \& Souberman, E. Harvard University Press.

Volterra, V. \& Erting, C. (1994/1990): From gesture to language in hearing and deaf children. Berlin: Springer-Verlag. (1994-2 ${ }^{\text {nd }}$ Edition, Washington, DC: Gallaudet University Press).

Werner, H. and Kaplan, B. (1963): Symbol formation. New York: Wiley. 